\title{
Efecto de mercurio sobre el transporte celular del agua en plantas. Una revisión
}

\section{Effect of mercury on celular transport of water in plants. A review}

JULIÁN FERNANDO CÁRDENAS-HERNÁNDEZ1,3

LIZ PATRICIA MORENO F.2

STANISLAV V. MAGNITSKIY²

\section{El mercurio interviene}

en el estado hídrico

de las plantas vía foliar.

Foto: G. Fischer

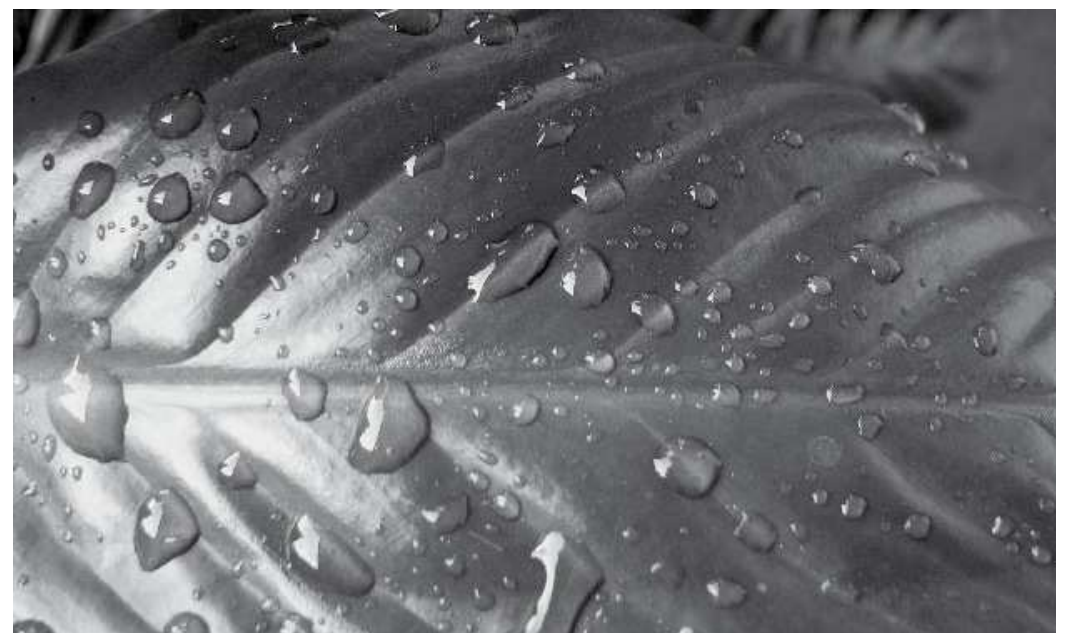

\section{RESUMEN}

Los metales pesados (MP) pueden afectar las relaciones hídricas de la planta de muchas formas. El Hg inhibe la actividad de las acuaporinas, proteínas que forman los canales transportadores de agua, y de esta manera afecta la conductividad a nivel celular y de tejidos en zonas específicas de la raíz, así como el volumen de las células guarda modificando los movimientos estomáticos. Además, junto con otros metales pesados, el $\mathrm{Hg}$ produce cambios morfológicos como el acortamiento o modificación en la elasticidad de las raíces, disminuyendo el área efectiva para la toma de agua. En hojas, el cambio en el número de estomas y de tricomas causado por el $\mathrm{Hg}$, altera la tasa de transpiración. En este artículo se revisa el efecto del Hg sobre el transporte de agua en la planta a nivel celular y su relación con el estatus hídrico de ésta. Además, se presentan avances recientes en el conocimiento de las acuaporinas basados en la utilización del Hg como inhibidor de su actividad.

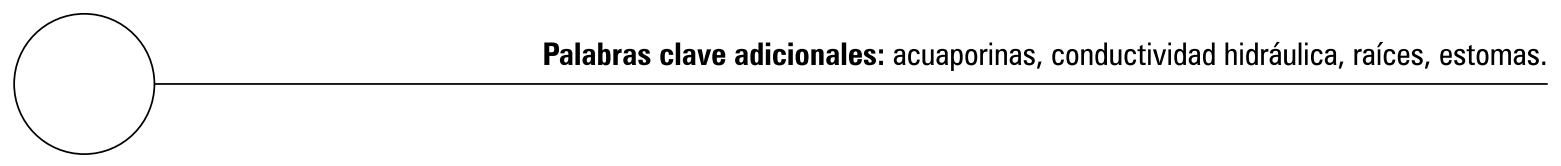

\footnotetext{
Programa de Maestría en Ciencias Agrarias, Facultad de Agronomía, Universidad Nacional de Colombia, Bogotá (Colombia).

2 Departamento de Agronomía, Facultad de Agronomía, Universidad Nacional de Colombia, Bogotá (Colombia).

3 Autor para correspondencia.jfcardenash@unal.edu.co
} 


\section{ABSTRACT}

Heavy metals could affect hydric relationships in plants in many ways. Mercury inhibits activity of aquaporins, the proteins that form the chanels-transporters of water and, thus, affects water conductivity of cells and tissues of special root zones as well as volume of guard cells modifying stomatal movements. Mercury produces morphological changes in plants, the effects typical for other heavy metals, such as shortening of root or decrease in root elasticity, thus reducing effective area for water absorption. In leaves, the changes in number of stomata and trichomes caused by the effects of $\mathrm{Hg}$ alter the transpiration rates. In the present article, the effects of $\mathrm{Hg}$ over the water transport in plants at cellular level and whole hydric status of plants are revised. Additionally, the recent studies on aquaporin functioning are discussed, with $\mathrm{Hg}$ used as an agent to inhibit aquaporin activity.

Additional key words: aquaporins, hydraulic conductivity, roots, stomata.

Fecha de recepción: 02-09-2009

Aprobado para publicación: 30-11-2009

INTRODUCCIÓN

Las plantas necesitan elementos esenciales para completar su ciclo de vida. Algunos de estos elementos son requeridos en concentraciones altas y son conocidos como macronutrientes, sin embargo, otros como el hierro, manganeso, molibdeno, cobre, zinc y níquel, llamados micronutrientes, son requeridos en cantidades mínimas (Arnon y Stout, 1939). Además las plantas absorben elementos que no tienen una función fisiológica conocida y que incluso son tóxicos en bajas concentraciones, tales como, el cromo, el mercurio y el plomo, conocidos como metales pesados (MP) (Mendelssohn et al., 2001; Zhang et al, 2001; Shaw et al., 2004; Burzynski y Zurek, 2007). Estos elementos alteran los procesos fisiológicos de la planta y pueden tener un efecto negativo en su crecimiento y desarrollo aunque también los elementos esenciales, especialmente los micronutrientes, pueden convertirse en tóxicos cuando se absorben sobre ciertos valores límite (Peralta-Videa et al., 2009). En Colombia, la contaminación del agua de riego con MP representa un problema importante, no solo por sus efectos visibles sobre plantas cultivadas, sino por sus efectos sobre la salud de los consumidores de dichos productos (Miranda et al., 2008).

Los $\mathrm{MP}$, incluido el mercurio $(\mathrm{Hg})$, disminuyen la tasa de crecimiento de las plantas debido a que afectan varios procesos del metabolismo radicular, causando inhibición de la toma de agua y nutrientes (Tamás et al., 2008), alteración del funcionamiento de las membranas (Hernández y Cooke, 1997), inhibición de la actividad enzimática (Tamás et al., 2006), oxidación y unión entre proteínas (Ortega-Villasante et al., 2005), inhibición de la división celular (Fusconi et al., 2006) y muerte celular (Ortega-Villasante et al., 2005). Estos efectos, producto de la toxicidad por MP, están asociados al estrés oxidativo producido por estos, tanto en raíces como en hojas (Ortega-Villasante et al., 2005; Romero-Puertas et al., 2004) que además causa la producción de peróxido de hidrógeno y un aumento del nivel de peroxidación de lípidos (Cho y Park, 2000; Dixit et al., 2000; Hao et al., 2006). 
Además de los efectos directos de los iones metálicos sobre la fisiología de la planta, su presencia puede volverla más susceptible a estreses como el déficit hídrico, debido a una menor capacidad de absorción de agua dada por un disminución en el desarrollo y funcionamiento del sistema radicular y posiblemente a un bloqueo de los canales de agua, esto va a causar una menor eficiencia en el uso del agua por parte de la planta (Yang et al., 2004; Ionenko et al., 2006; Ryser y Emerson, 2007). Los efectos de los MP y especialmente del $\mathrm{Hg}$ sobre los procesos fisiológicos que determinan la regulación hídrica de la planta han sido observados frecuentemente (Poschenrieder y Barceló, 2004; Santala y Ryser, 2009).

En numerosos estudios se ha reportado el marchitamiento de las plantas como consecuencia de la toxicidad por MP y por esto ha sido ampliamente investigado el efecto de estos elementos sobre las pérdidas de agua y el comportamiento estomático de diferentes plantas (Perfus-Barbeoch et al., 2002; Gupta y Sinha, 2009). El efecto de las concentraciones de metales pesados entre $0,2 \mathrm{y}$ $1 \mathrm{mM}$ sobre el movimiento de los estomas y el mecanismo como este puede darse es aún objeto de investigacion (Yang et al., 2004).

Es probable que metales pesados como $\mathrm{Hg}$ afecten los movimientos estomáticos por la inhibición de los canales de agua y limiten el flujo de esta dentro de las células guarda (Singh y Sinha, 2004). Una red de canales iónicos, la cual puede controlar el movimiento de los estomas, ha sido bien caracterizada en las membranas plasmática y vacuolar de las células guarda (Allen et al., 2001; Schroeder et al., 2001 y Zhang et al., 2001). En el plasmalema y el tonoplasto de las células guarda se han encontrado canales de agua, los cuales permiten de forma rápida y específica su transporte. Ciertas concentraciones de magnesio y calcio inhiben los canales de agua y esto ocasiona cambios en los movimientos estomáticos (Gerbeau et al., 2002). El Hg produce el mismo efecto, lo cual comprueba la participación de los canales de agua en el movimiento estomático y su inhibición por este elemento (Yang et al., 2002). Se ha encontrado que el $\mathrm{HgCl}_{2}$ puede inhibir los canales de agua en la planta para regular el transporte de agua (Clarkson et al., 2000). En el presente artículo se presenta información reciente sobre el efecto del $\mathrm{Hg}$ en la función de las acuaporinas en las plantas y en la conductividad hidráulica en la raíz y las hojas con el fin de dilucidar algunos aspectos relacionados con el efecto del $\mathrm{Hg}$ en el estatus hídrico de las plantas.

\section{Acuaporinas y su papel en el transporte de agua en las plantas}

Las acuaporinas son proteínas de las membranas intracelulares y plasmáticas que se encuentran dentro del grupo de las denominadas proteínas intrínsecas mayores, las cuales están encargadas de facilitar el transporte de agua a través de membranas biológicas en todo tipo de organismos como bacterias, animales y plantas (Zhang et al., 2008). Las acuaporinas son vías proteicas transmembranales no solo para el agua (Quigley et al., 2001), sino para algunos pequeños solutos sin carga (Gerbeau et al., 1999), $\mathrm{CO}_{2}$ (Endevard et al., 2006) y ácido bórico (Maurel et al., 2008).

El descubrimiento de las acuaporinas se dio en 1988 cuando Peter Agre y su equipo, de la Universidad Johns Hopkins, estudiaban las proteínas de la membrana de los eritrocitos. Durante sus trabajos de purificación de la proteína de 32 kilodalton que determina el grupo sanguíneo $\mathrm{Rh}$, encontraron un polipéptido de peso molecular inferior $(28 \mathrm{kDa})$ que copurificaba con la proteína de interés. Después de continuar con las investigaciones encontraron que se trataba de una nueva proteína integral de membrana. Inicialmente la denominaron CHIP28, posteriormente recibiría el nombre de AOP1, la función de esta proteína fue determinada hasta 1992 (Echeverría y Zardoya, 2006).

Los investigadores hallaron que algunas células animales inyectadas con cantidades pequeñas de ARN mensajero de AQP1 desarrollaban 
una permeabilidad al agua superior a las células control sin inyectar o inyectadas con agua. Se descubrió también que la permeabilidad al agua dependiente de AQP1 se inhibía con cloruro de mercurio y que tal efecto se revertía con agentes reductores (Echeverría y Zardoya, 2006). Este comportamiento correspondía a un flujo de agua mediado por canales, lo que significaba que habían descubierto una proteína que funcionaba como un canal de agua. Este descubrimiento ha permitido explicar mejor algunos procesos celulares y características de tejidos en animales y plantas (Echeverría y Zardoya, 2006).

Las acuaporinas en las plantas han sido clasificadas en cuatro grandes grupos: proteínas intrínsecas de la membrana plasmática (PIP) con dos subgrupos filogenéticos PIP1 y PIP2; proteínas intrínsecas del tonoplasto (TIP); nodulinas intrínsecas (NIP) donde NOD26 es una acuaporina descubierta en la membrana peribacterial de las raíces noduladas de soya; y pequeñas proteínas básicas intrínsecas (SIP) que se han observado en la membrana del retículo endoplasmático (Ishikawa et al., 2005).

La primera evidencia del papel de las acuaporinas en la toma de agua a nivel celular y en el transporte de esta en toda la planta surgió de plantas transgénicas antisentido para PIP, las cuales desarrollaron un sistema radicular más grande que las plantas control (Kaldenhoff et al., 1998). En tabaco, la acuaporina de la membrana plasmática NtAQP1 mostró ser importante para la conductividad hidráulica y la resistencia al estrés hídrico (Siefritz et al., 2002). Los estudios en plantas con producción desigual de $\mathrm{P} 1 \mathrm{P} 1$ y $\mathrm{P} 1 \mathrm{P} 2$ indicaron que ambas acuaporinas son necesarias para la recuperación del défici hídrico (Martre et al., 2002). Las acuaporinas no solo controlan el transporte de agua de las raíces a las hojas en la corriente transpiratoria, sino que regulan otros procesos como el transporte de asimilados en el floema, la apertura y cierre de los estomas en las hojas, el movimiento de las hojas y el control de la homeostasis citoplasmática (Chaumont et al., 2005).
Existen dos vías posibles para el flujo osmótico del agua entre los tejidos de las plantas: la vía apoplástica conformada por las paredes celulares, los espacios intercelulares y el xilema y la vía simplástica conformada por el continuo citoplasmático y la vacuola (transporte a través de membranas y plasmodesmata). El agua se mueve, todo el tiempo, utilizando simultáneamente las dos vías. La vía más utilizada depende de la especie, el órgano, la condición fisiológica de la planta al igual que la fuerza conductora (hidrostática o presión osmótica). La presencia de acuaporinas en el tonoplasto aumenta la efectividad en el transporte del agua, una vez esta ha atravesado la membrana celular (Chrispeels y Maurel, 1994).

El flujo de agua a través de las células se puede modificar alterando el comportamiento individual de los canales de agua o la abundancia de los mismos en las membranas. Cuando hay poca regulación de las acuaporinas en el tonoplasto la conductividad hidráulica puede disminuir. Para aumentar la conductividad transcelular del agua, la célula puede aumentar el número de acuaporinas en el plasmalema, a través del aumento de la expresión de los genes que codifican algunas PIP, esto puede ocurrir durante el estrés por déficit hídrico (Chrispeels y Maurel, 1994).

\section{Efecto del mercurio sobre las acuaporinas}

Recientemente se han desarrollado herramientas específicas para monitorear la expresión de la familia entera de las acuaporinas. Utilizando librerías de cDNA e hibridándolas con secuencias específicas de genes de las acuaporinas se ha encontrado una regulación coordinada de estos genes en respuesta a estrés por déficit hídrico y por nutrientes (Maathuis et al., 2003; Alexandersson et al., 2005). Los análisis cuantitativos por RTPCR han sido usados para establecer la abundancia de los transcriptos de las acuaporinas en varios tejidos y órganos bajo diferentes condiciones de estrés (Jang et al., 2004; Alexandersson et al., 2005; Hachez et al., 2006; Sakurai et al., 2005). También se ha desarrollado un mapeo detalla- 
do de la expresión celular específica de los genes de las acuaporinas en brotes de maíz y raíces de Arabidopsis thaliana basado en el análisis de RTPCR in situ (Maurel, 2007).

El alto número de aminoácidos idénticos entre homólogos cercanos de acuaporinas (más del 97\%) hace muy dispendiosa la inmunodetección específica de una simple isoforma de acuaporina. Sin embargo, anticuerpos de reacción cruzada con miembros de subclases específicas de acuaporinas de plantas han mostrado ser útiles para caracterizar la expresión en varios tipos celulares y órganos (Kobae et al., 2006; Vander Willigen et al., 2006). Debido a su relativa alta abundancia en las membranas de las plantas, y a su marcado carácter hidrófobo, las acuaporinas se pueden analizar fácilmente mediante espectrometría de masas (Santoni et al., 2006). Esta técnica de alta resolución puede distinguir entre acuaporinas homólogas muy cercanas, razón por la cual permite realizar un seguimiento exacto de las acuaporinas presentes (Maurel, 2007).

Se ha encontrado que el transporte de $\mathrm{Hg}$ y otros MP está directamente relacionado con el flujo de agua en las plantas. En $A$. thaliana L. se encontró que la proteína asociada a MP (AtHMA3) pertenece al subgrupo $\mathrm{P}_{1 \mathrm{~B}-2}$ de la familia de las ATPasas tipo P, las cuales están involucradas en el transporte de MP. Estas proteínas se encuentran localizadas en el tonoplasto con un alto nivel de expresión en células guarda, hidátodos, tejido vascular y el ápice de la raíz (Morel et al., 2009).

Las acuaporinas en las células guarda pueden estar involucradas en los movimientos estomáticos (Sun et al., 2001; Huang et al., 2002; López-Berenguer et al., 2007; Ehlert et al., 2009). Varios experimentos han demostrado que los compuestos de $\mathrm{Hg}$ inhiben los canales de agua de las plantas a concentraciones submilimolares, pero existen algunas excepciones (Yang et al., 2004). En células animales, el $\mathrm{Hg}$ inhibe las acuaporinas y se ha identificado el aminoácido Cys ${ }^{189}$ como el sitio de inhibición del Hg de AQP1. Por otro lado
AQP4 ha sido considerada como una acuaporina insensible al $\mathrm{Hg}$ ya que esta no posee el residuo reactivo de cisteína correspondiente a Cys ${ }^{189} \mathrm{de}$ AOP1 (Yukutake et al., 2008).

El estudio reciente de las acuaporinas ha demostrado que el $\mathrm{Hg}$ interfiere en la actividad de las mismas, y esto ha permitido avanzar en el estudio de su comportamiento e influencia sobre características de conductividad hidráulica y movimiento del agua (Echeverría y Zardoya, 2006).

\section{Acuaporinas en la raíz y su efecto sobre la conductividad hidráulica}

Un gran número de acuaporinas se expresan en raíces (Bramley et al., 2007). Estas proteínas integrales de la membrana forman canales conductores de agua, los cuales son considerados responsables de la variación en la conductividad hidráulica en los sistemas de raíces (Javot y Maurel, 2002).

Utilizando la genética inversa se ha demostrado que las acuaporinas están relacionadas con el estatus hídrico de algunas especies durante un estrés abiótico (Yu et al., 2005; Jang et al., 2007), sin embargo, es posible que otros transportadores estén involucrados en la osmorregulación. Todo esto en conjunto puede causar cambios en la morfología del sistema radicular. Por ejemplo, la mayor masa radicular de mutantes de $A$. thaliana y Nicotina tabacum parece ser un efecto compensatorio de la poca expresión de las acuaporinas de la membrana plasmática que reduce la permeabilidad de algunas células (Martre et al., 2002; Siefritz et al., 2002; Bramley et al., 2009).

Los canales de agua sensibles a $\mathrm{Hg}$ parecen estar involucrados en la disminución de la conductividad hidráulica desde la raíz hasta las hojas. Se ha demostrado que los iones de $\mathrm{Hg}$ inhiben rápidamente el transporte de agua a través de las raíces aisladas de plantas de cereales como maíz y trigo. Adicionalmente, se comprobó que la presencia de $\mathrm{Hg}$ en las raíces reduce la conductancia 
hidráulica y además puede limitar el crecimiento de las raíces que están sometidas a estrés hídrico (Lu y Neumann, 1999).

Un estudio sobre la conductividad hidráulica de Lupinus angustifolius L., Lupinus luteus L. y Triticum aestevium L. demostró un papel principal de la estructura radicular y la anatomía de la raíz sobre el transporte de agua, así como la influencia de las acuaporinas. A partir de los valores de conductividad hidráulica celular alta y la fuerte inhibición por Hg se determinó que la actividad de las acuaporinas es constante en todas las células del cortex y epidermis de las tres especies (Bramley et al., 2009). La conductividad hidráulica de raíces individuales y grupos de raíces de $T$. aestivum se redujo a más de la mitad después de ser tratadas con $\mathrm{Hg}$. Sin embargo, a nivel celular, en L. angustifolius la conductividad hidráulica de las células de las raíces tratadas se redujo en $33 \%$, en L. luteus en $86 \%$ y en T. aestivum en $77 \%$ (Bramley et al., 2009).

Las capas de células más externas de las raíces de L. angustifolius fueron particularmente sensibles al tratamiento con $\mathrm{Hg}$. Si el flujo de agua ocurre completamente a través de las células, cruzando membranas, la inhibición de la conductividad hidráulica debería reflejarse también en la conductividad de toda la raíz. Sin embargo, a nivel de sistema radicular, el control por parte de las acuaporinas del flujo del agua estuvo limitado a pequeñas regiones de la endodermis en el trigo, mientras que en lupino parece ser que el flujo del agua ocurrió predominantemente por la vía apoplastica sin ser influenciado por las acuaporinas (Bramley et al., 2009).

Consistente con lo anterior, al evaluar el efecto del Hg sobre las raíces del maíz se encontró que la conductividad hidráulica celular de las células cercanas al ápice no fue afectada por el tratamiento de $\mathrm{Hg}$, en contraste con las células más maduras en fase de crecimiento, donde la conductividad hidráulica se vió fuertemente reducida. A pesar que la concentración de $\mathrm{Hg}$ fue relativamente baja, $20 \mathrm{mM}$ de $\mathrm{HgCl}_{2}$, las zonas sensibles se vieron muy afectadas en la toma de agua y presentaron cambios en la pared celular (Hukin et al., 2002).

Una reducción en el crecimiento causado por un cambio en la conductividad hidráulica se esperaría que produjera un mayor gradiente de potencial hídrico en la región afectada (Boyer, 1985). En raíces de maíz tratadas con $\mathrm{Hg}$, durante los primeros 10 min del tratamiento el turgor se redujo en las células en crecimiento ubicadas entre los 5 y $7 \mathrm{~mm}$ del extremo apical de la raíz, causando una disminución en el potencial hídrico pero sin cambios en la presión osmótica. En contraste, no se reportaron cambios en el potencial hídrico de las células en crecimiento a $3 \mathrm{~mm}$ ni en células de la región de no crecimiento ubicada entre los 12 y $20 \mathrm{~mm}$. Se desconcoce si la correlación existente entre la conductividad hidráulica y el crecimiento es o no cuantitativa (Hukin et al., 2002).

Mediante el análisis de RT-PCR, se encontró que existía un mayor nivel de expresión de los genes de las proteínas intrínsecas del plasmalema, ZmPiP1-2 y ZmPiP2-4 en las zonas jóvenes en crecimiento que en las zonas viejas y mucho mayor que en el ápice de la raíz. Sin embargo, el gen de la acuaporina del tonoplasto (ZmTIP1-1) mostró igual nivel de expresión en ambas regiones de la zona de crecimiento (Hukin et al., 2002).

Por otro lado, al aplicar el compuesto fluorescente carboxifluoresceina, se demostró que existe conexión simplásmica entre el floema y las células corticales a $3 \mathrm{~mm}$ de la punta de la raíz pero esto no se da en la raíz de los $5-20 \mathrm{~mm}$ de la punta. Esto es consistente con la disminución en la continuidad simplásmica a lo largo de la zona de crecimiento y evidencia un cambio en la vía principal del agua durante el desarrollo de la zona de crecimiento de las células radiculares (Hukin et al., 2002).

Barrowclough et al. (2000) demostraron que la sensibilidad de la conductividad hidráulica a $\mathrm{Hg}$ 
cambia a lo largo de la raíz, sin embargo, la región en la que no se observan cambios significativos es la correspondiente a las células viejas y no a las jóvenes, contrario a lo que afirman Hukin et al. (2002). Esto parece aclararse al tener en cuenta que Barrowclough et al. (2000) denominaron como células jóvenes a las que se encuentran en la zona comprendida entre los 30 y $40 \mathrm{~mm}$ después de la punta de la raíz, mientras para $\mathrm{Hu}-$ kin et al. (2002) esta denominación se refiere a una zona entre 5 y $12 \mathrm{~mm}$. Barrowclough et al. (2000) atribuyen este comportamiento a la formación de barreras endo y exodérmicas que previnieron la entrada de $\mathrm{Hg}$ al tejido. Sin embargo, según Zimmermann et al. (2000) tales barreras apoplásticas usualmente no se presentan en raíces de maíz en hidroponía, condición en la que se encontraban las plantas del estudio.

El incremento en la sensibilidad a $\mathrm{Hg}$ a lo largo de la zona de crecimiento radicular estuvo acompañado de cambios en la conexión simplástica de las células. Las células a $3 \mathrm{~mm}$ estuvieron asociadas a nivel de simplasto a través del perfil radial de la raíz. Esta conexión va disminuyendo a lo largo de la raíz y se observa que las células se aíslan a los $5 \mathrm{~mm}$, mientras en células de mayor edad entre los 5 y $20 \mathrm{~mm}$ la fluorescencia estuvo confinada al floema. La disminución uniforme de la continuidad simplástica durante el desarrollo también se reportó en raíces de A. thaliana ( $\mathrm{Zhu}$ et al., 1998); en fríjol se reportó que una conexión simplástica similar parece estar confinada a la región apical de la zona de elongación (Patrick y Offler, 1996). En raíces de maíz, la longitud de la zona donde predomina el transporte simplástico depende de la cantidad de solutos disponibles en la raíz y estuvo regulada aparentemente por la apertura y cierre de los plasmodesmata (Hukin et al., 2002).

Lo anterior indica que las regiones donde la conductividad hidráulica es sensible a Hg coinciden con las regiones de aislamiento simplástico, sugiriendo que los sitios donde no existe la vía simplástica para la toma de agua, es necesario un flujo de agua a través de las membranas y esto coincide con la presencia de acuaporinas funcionales en esta región (Hukin et al., 2002).

El mercurio además de afectar el comportamiento de algunos canales hídricos también afecta la toma de nutrientes necesarios para el balance hídrico. Los altos niveles de $\mathrm{Hg}$ resultan en niveles más bajos de $\mathrm{K}, \mathrm{Mn}$ y $\mathrm{Mg}$ tanto en raíces como en brotes, encontrándose también acumulaciones de Fe en los ápices radiculares. Los cambios promovidos por $\mathrm{Hg}$ son mayores en los ápices que en partes más viejas de las raíces. Por otro lado, se reporta un alto incremento de la toma de ${ }^{45} \mathrm{Ca}$ en presencia de $\mathrm{HgCl}_{2}$ (Godbold, 1991), esto puede deberse a que el $\mathrm{Hg}$ es tomado en lugar de otros nutrientes (Patra et al., 2004). Además se debe considerar el hecho que $\mathrm{Hg}$ denatura las proteínas reduciendo el funcionamiento de algunas enzimas y de transportadores de Py K (Patra et al., 2004; Du et al., 2005; Moreno-Jimenez et al., 2007).

\section{Acuaporinas en las hojas y su influencia sobre los movimientos estomáticos}

$\mathrm{El} \mathrm{Hg}$, al igual que otros MP, afecta notablemente los movimientos estomáticos a concentraciones submilimolares, probablemente de diferentes formas. El cloruro de lantano $\mathrm{LaCl}_{3}$, que bloquea los canales de calcio, aparentemente afecta los cambios en las concentraciones del Ca citosólico en las células guarda, lo cual influye indirectamente en la actividad de otros canales iónicos, tales como los canales de $\mathrm{K}, \mathrm{Cl}$ y malato y finalmente la apertura y cierre estomático (Yang et al., 2002).

La inhibición del flujo de agua a través de los canales por las bajas concentraciones de $\mathrm{Hg}$ reactivo se ha evaluado en muchos experimentos (Yang et al., 2004; Clarkson et al., 2000; Daniels et al., 1996). En cortes de epidermis abaxial de Vicia faba L., cv. Dabaican incubados con diferentes concentraciones de $\mathrm{HgCl}_{2}, \mathrm{ZnCl}_{2}, \mathrm{PbCl}_{2}$, $\mathrm{LaCl}_{3}, \mathrm{KCl}, \mathrm{NaCl}$, y $\mathrm{MgCl}_{2}$ se encontró que el 
$\mathrm{HgCl}_{2}$ afectó el movimiento estomático principalmente por el bloqueo de los canales de agua, presentándose diferencias significativas en el movimiento estomático en las plantas sometidas a este tratamiento, al igual que en las tratadas con $\mathrm{LaCl}_{3}$. Lo anterior puede ser explicado teniendo en cuenta que el $\mathrm{HgCl}_{2}$ inhibe los canales de agua en hojas de Vicia faba (Huang et al., 2002; Yang, 2002). Los efectos principales causados por concentraciones altas de $\mathrm{HgCl}_{2}$ han sido interpretados como toxicidad por $\mathrm{Hg}$, pero las bajas concentraciones pueden causar efectos directos o indirectos sobre los canales de agua (Lu y Neumann, 1999; Yang et al., 2002, 2004). Algunos investigadores consideran que el $\mathrm{Hg}$ puede alterar el metabolismo o causar la depolarización de la membrana, lo cual podría afectar de diferente forma los movimientos de las células guarda. Sin embargo, se ha encontrado que al aplicar mercaptoetanol, un agente reductor, se revierte la inhibición del movimiento estomático causada por $\mathrm{HgCl}_{2}$. Adicional a esto, incluso cuando la duración de la exposición no fue muy larga el $\mathrm{HgCl}_{2}$ produjo efectos leves sobre otros canales y enzimas (Yang et al., 2004).

El efecto del $\mathrm{HgCl}_{2}$ fue específico sobre los canales de agua, mientras que el $\mathrm{LaCl}_{3}$ afectó la mayoría de canales iónicos en las membranas. Como resultado, el potencial osmótico y el potencial hídrico se vieron afectados en el interior y en el exterior de las células guarda, el flujo de agua fue limitado y el volumen de las células guarda no cambió. Con el tiempo, la inhibición de los movimientos estomáticos disminuyó tanto a plena luz como en oscuridad. Esto se debe a que el agua se mueve a través de la matriz lipídica de la membrana aun cuando los canales de agua esten inhibidos y el volumen de las células guarda se modificó y por tanto su apertura (Yang et al., 2004).

La cantidad de estomas abiertos sobre el total nunca alcanzó una proporción de 1 o de 0 en el estudio realizado por Yang et al. (2004) donde tampoco se inhibieron los movimientos esto- máticos completamente por los tratamientos de $\mathrm{HgCl}_{2}, \mathrm{LaCl}_{3}$ y $\mathrm{HgCl}_{2}+\mathrm{LaCl}_{3}$. No todos los estomas estaban en el mismo estado, algunos aparecían abiertos, mientras otros permanecían cerrados. Las plantas pueden adaptarse a su ambiente o responder al estrés mediante la regulación celular de sus relaciones hídricas a través de los canales hídricos (López-Berenguer et al., 2007; Netting, 2000 y Clarkson et al., 2000).

Otros MP como el Pby el Zn también causaron efectos significativos sobre el movimiento estomático y la inhibición fue más obvia a medida que aumentaba la concentración. La apertura estomática después de la incubación con $\mathrm{Pb}$ y $\mathrm{Zn}$ mostró un comportamiento similar al presentado después de la incubación con $\mathrm{Hg}$, las hojas presentaron menor cantidad de estomas abiertos durante el día y mayor durante la noche, en comparación con los tratamientos con $\mathrm{K}, \mathrm{Na}, \mathrm{Mg}$ y el testigo. Los iones del $\mathrm{Pb}$, el Zn y el Hg tienen diámetros atómicos y valencias similares y además comparten los mismos mecanismos para afectar los canales de agua (Yang et al., 2004).

El efecto directo del $\mathrm{Hg}$ sobre los canales de transporte aumenta los efectos causados por la alta toxicidad del metal. En raíces, al igual que en hojas, la alta toxicidad de $\mathrm{Hg}$ se relaciona con la fuerte inhibición de los canales de agua por $\mathrm{HgCl}_{2}$ que causan estrés hídrico, que puede ocasionar además un incremento en el estrés oxidativo inducido por Hg (Zhang y Tyerman, 1999).

\section{CONCLUSIÓN}

Existen numerosos factores abióticos que causan estrés hídrico en plantas, sin embargo las plantas han desarrollado estrategias para superar esta condición modificando los mecanismos de transporte de agua, tanto a nievel simplástico como apoplástico. Debido a que las acuaporinas son canales de agua que permiten el transporte eficiente de ésta a través de las membranas 
evitando su paso a través del núcleo lipídico, cualquier factor que afecte su funcionamiento modifica el estatus hídrico de la planta. El Hg $y$ algunos otros MP pueden inhibir a las acuaporinas y de esta manera disminuir la capacidad de respuesta de la planta ante una condición de déficit hídrico. Además, este elemento afecta la morfología de las raíces y el número de estomas en las hojas lo cual modifica la relación entre la toma de agua y su pérdida por el proceso de transpiración. El problema actual de disponibilidad de aguas de calidad para riego hace frecuente el uso de aguas con alto contenido de MP, incluido el $\mathrm{Hg}$. La presencia de $\mathrm{Hg}$ en la planta, aún en bajas concentraciones, afecta directamente el funcionamiento de las acuaporinas, además de afectar varios procesos metabólicos. Dado su efecto, es importante tener claro el papel del $\mathrm{Hg}$ ya que como resultado del cambio climático, que ya ha comenzado a afectar de manera directa la producción vegetal, las plantas tienen que desarrollarse bajo condiciones limitadas de agua $y$, por tanto, necesitan mejorar los mecanimos de transporte de esta. De otro lado, el $\mathrm{Hg}$ dada su capacidad de inhibir a las acuaporinas, ha sido una herramienta valiosa en la caracterización de la función e importancia de estas como transportadoras de agua bajo diferentes condiciones, incluidas las ocasionadas por el estrés abiótico. Esta caracterización ha permitido determinar que la manipulación genética de las acuaporinas es un mecanismo para obtener plantas con un uso más eficiente del agua y una mejor respuestas a condiciones de déficit hídrico.

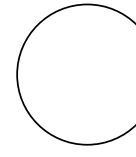

Alexandersson, E.; L. Fraysse; S. Sjovall-Larsen; S. Gustavsson; M. Fellert; M. Karlsson; U. Johanson y P. Kjellbom. 2005. Whole gene family expression and drought stress regulation of aquaporins. Plant Mol. Biol. 59, 469-484.

Allen, G.J.; S.P. Chu; C.L. Harrington; K. Schumacher; T. Hoffmann; Y.Y. Tang; E. Gill y J.I. Schroeder. 2001. A defined range of guard cell calcium oscillation parameters encodes stomatal movements. Nature 411, 1053-1057.

Arnon, D.I. y P.R. Stout. 1939. The essentially of certain elements in minute quantity for plants with special reference to copper. Plant Physiol.14, 371-375.

Barrowclough D.E.; C.A. Peterson y E. Steudle. 2000. Radial hydraulic conductivity along developing onion roots. J. Exp. Bot. 344, 547-557.

Boyer, J.S. 1985. Water transport. Annu. Rev. Plant Physiol. $36,473-516$

Bramley, H.; N.C. Turner; D.W. Turner y S.D. Tyerman. 2009. Roles of morphology, anatomy, and aquaporins in determining contrasting hydraulic behavior of roots. Plant Physiol. 150, 348-364.

Bramley, H.; D.W. Turner; S.D. Tyerman y N.C. Turner. 2007.Water flow in the roots of crop species: the

\section{REFERENCIAS BIBLIOGRÁFICAS}

influence of root structure, aquaporin activity, and waterlogging. Adv. Agron. 96, 133-196

Burzynski, M. y A. Zurek. 2007. Effects of copper and cadmium on photosynthesis in cucumber cotyledons. Photosynthetica 45, 239-244.

Chaumont, F. M. Moshelion y M.J. Daniels. 2005. Regulation of plant aquaporin activity. Biol. Cell. 97(10), 749-764.

Cho, U. y J. Park. 2000. Mercury-induced oxidative stress in tomato seedlings. Plant Sci. 156, 1-9.

Chrispeels, M.J. y C. Maurel. 1994. Aquaporins: the molecular basis of facilitated water movement through living plant cells. Plant Physiol. 105, 9-13.

Clarkson, D.T.; M. Carvajal; T. Henzler; R.N. Waterhouse; A.J. Smyth; D.T. Cooke y E. Steudle. 2000. Root hydraulic conductance: Diurnal aquaporin expression and the effects of nutrient stress. J. Exp. Bot. 51, 61-70.

Daniels, M.J.; F.Chaumont; T.E. Mirkov y M.J. Chrispeels. 1996. Characterization of a new vacuolar membrane aquaporin sensitive to mercury at a unique site. Plant Cell 8, 587-599.

Dixit, V.; V. Pandey y R. Shyam. 2000. Differential antioxidative responses to cadmium in roots and leaves 
of pea (Pisum sativum L. cv. Azad). J. Exp. Bot. 52, 1101-1109.

Du, X.; Y.G. Zhu; W.J. Liu y X.S. Zhao. 2005. Uptake of mercury $(\mathrm{Hg})$ by seedlings of rice (Oryza sativa L.) grown in solution culture and interactions with arsenate uptake. Environ. Exp. Bot. 54, 1-7.

Ehlert, C.; C. Maurel; F. Tardieu y T. Simonneau. 2009. Aquaporin-mediated reduction in maize root hydraulic conductivity impacts cell turgor and leaf elongation even without changing transpiration. Plant Physiol. 150, 1093-1104.

Endeward, V.; R. Musa-Aziz; G.J. Cooper; L.M. Chen; M.F. Pelletier; L.V. Virkki; C.T. Supuran, L.S. King, W. F. Boron y G. Gros. 2006. Evidence that aquaporin 1 is a major pathway for $\mathrm{CO}_{2}$ transport across the human erythrocyte membrane. FASEB J. 20, 1974-1981.

Echeverría, M. y R. Zardoya. 2006. Acuaporinas: los canales de agua celulares. Investig. Ciencia 363, 60-67.

Fusconi, A.; O. Repetto; E. Bona; N. Massa; C. Gallo; E. Dumas-Gaudot y G. Berta.2006. Effects of cadmium on meristem activity and nucleus ploidy in roots of Pisum sativum L. cv. Frisson seedlings. Environ. Exp. Bot. 58, 253-260.

Gerbeau, P.; G. Amodeo; T. Henzler; V. Santoni; P. Ripoche y C. Maurel. 2002. The water permeability of Arabidopsis plasma membrane is regulated by divalent cations and pH. Plant J. 30, 71-81.

Gerbeau, P.; J. Guclu; P. Ripoche y C. Maurel. 1999. Aquaporin Nt-TIPa can account for the high permeability of tobacco cell vacuolar membrane to small neutral solutes. Plant J. 18, 577-587.

Godbold, D.L. 1991. Mercury-induced root damage in spruce seedlings. Water Air Soil Pollut. 56, 1.

Gupta A.K. y S. Sinha. 2009. Antioxidant response in sesame plants grown on industrially contaminated soil: Effect on oil yield and tolerance to lipid peroxidation. Bioresour. Technol. 100, 179-185.

Hachez, C.; M. Moshelion; E. Zelazny; D. Cavez y F. Chaumont. 2006. Localization and quantification of plasma membrane aquaporin expression in maize primary root: a clue to understanding their role as cellular plumbers. Plant Mol. Biol. 62, 305-323.

Hao, F.; X. Wang y J. Chen. 2006. Involvement of plasmamembrane NADPH oxidase in nickel-induced oxidative stress in roots of wheat seedlings. Plant Sci. 170, 151-158.

Hernández, L.E. y D.T. Cooke. 1997. Modification of the root plasma membrane lipid composition of cadmium-treated Pisum sativum. J. Exp. Bot. 48, 1375 1381.
Huang, R.F; M.J. Zhu; Y. Kang; J. Chen y X.C. Wang. 2002. Identification of plasma membrane aquaporin in guard cells of Vicia faba and its role in stomatal movement. Acta Bot. Sinica. 44, 42-48.

Hukin, D.; C. Doering-Saad; C.R. Thomas y J. Pritchard. 2002. Sensitivity of cell hydraulic conductivity to mercury is coincident with symplasmic isolation and expression of plasmalemma aquaporin genes in growing maize roots. Planta 215, 1047-1056.

Ionenko, I.F.; A.V. Anisimov y F.G. Karimova. 2006. Water transport in maize roots under the influence of mercuric chloride and water stress: a role of water channels. Biol. Plant. 50, 74-80.

Ishikawa, F; S. Suga; T. Uemura; M.H. Sato y M. Maeshima. 2005. Novel type aquaporin SIPs are mainly localized to the ER membrane and show cell-specific expression in Arabidopsis thaliana. FEBS Letters $579,5814-5820$.

Jang, J.; S. Lee; J. Rhee; G. Chung; S. Ahn y H. Kang. 2007. Transgenic Arabidopsis and tobacco plants overexpressing an aquaporin respond differently to various abiotic stresses. Plant Mol. Biol. 64, 621-632.

Jang, J.Y.; D.G. Kim; Y.O. Kim; J.S. Kim y H. Kang. 2004. An expression analysis of a gene family encoding plasma membrane aquaporins in response to abiotic stresses in Arabidopsis thaliana. Plant Mol. Biol. 54, 713-725

Javot, H. y C. Maurel. 2002. The role of aquaporins in root water uptake. Ann. Bot. 90, 301-313.

Kaldenhoff, R.; K. Grote; J.J. Zhu y U. Zimmermann. 1998. Significance of plasmalemma aquaporins for water-transport in Arabidopsis thaliana. Plant J. 14, 121-128.

Kobae, Y.; M. Mizutani; S. Segami y M. Maeshima. 2006. Immunochemical analysis of aquaporin isoforms in Arabidopsis suspension-cultured cells. Biosci. Biotechnol. Biochem. 70, 980-987.

López-Berenguer, C.; M. Martínez-Ballesta; C. GarcíaViguera y M. Carvajal. 2007. Leaf water balance mediated by aquaporins under salt stress and associated glucosinolate synthesis in broccoli. Plant Sci. 174, 321-328.

Lu, Z. y P.M. Neumann. 1999. Water stress inhibits hydraulic conductance and leaf growth in rice seedlings but not the transport of water via mercury-sensitive water channels in the root. Plant Physiol.120, 143151.

Maathuis, F.J.; V. Filatov; P. Herzyk; G.C. Krijger; K.B. Axelsen; S. Chen; B.J. Green; Y. Li; K.L. Madagan; R. Sánchez-Fernández; B.G. Forde; M.G. Palmgren; P.A. Rea; L.E. Williams; D. Sanders y A. Amtmann. 2003. Transcriptome analysis of root transporters 
reveals participation of multiple gene families in the response to cation stress. Plant J. 35, 675-692.

Martre, P.; R. Morillon; F. Barrieu; G.B. North; P.S. Nobel y M.J. Chrispeels. 2002. Plasma membrane aquaporins play a significant role during recovery from water deficit. Plant Physiol. 130, 2101-2110.

Maurel, C. 2007. Plant aquaporins: Novel functions and regulation properties. FEBS Letters 581, 2227-2236.

Maurel, C.; L. Verdoucq; D.T. Luu y V. Santoni. 2008. Plant aquaporins: membrane channels with multiple integrated functions. Annu. Rev. Plant Biol. 59, 595-624.

Mendelssohn, I.A.; K.L. McKee y T. Kong. 2001. A comparison of physiological indicators of sublethal cadmium stress in wetland plants. Environ. Exp. Bot. 46, 263-275.

Miranda, D.; C. Carranza y G. Fischer. 2008. Calidad del agua de riego en la Sabana de Bogotá. Facultad de Agronomía, Universidad Nacional de Colombia, Bogotá.

Morel, M.; J. Crouzet; A. Gravot; P. Auroy; N. Leonhardt; A. Vavasseur y P. Richaud. 2009. AtHMA3, a $\mathrm{P}_{1 \mathrm{~B}}$ ATPase Allowing Cd/Zn/Co/Pb Vacuolar Storage in Arabidopsis. Plant Physiol. 149, 894-904.

Moreno-Jimenez, E.; J.M. Penalosa; E. Esteban y R.O. Carpena-Ruiz. 2007. Mercury accumulation and resistance to mercury stress in Rumex induratus and Marrubium vulgare grown in perlite. J. Plant Nutr. Soil Sci. 170, 485-494.

Netting, A.G. 2000. pH, abscisic acid and the integration of metabolism in plants under stressed and nonstressed conditions: cellular responses to stress and their implication for plant water relations. J. Exp. Bot. 51, 147-158.

Ortega-Villasante, C.; R. Rellán-Álvarez; F.F. DelCampo; R.O. Carpena-Ruiz y L.E. Hernández. 2005. Cellular damage induced by cadmium and mercury in Medicago sativa. J. Exp. Bot. 56, 2239-2251.

Patra, M.; N. Bhowmik; B. Bandopadhyay y A. Sharma. 2004. Comparison of mercury, lead and arsenic with respect to genotoxic effects on plants systems and the development of genetic tolerance. Environ. Exp. Bot. 52, 199-223.

Patrick, J.W. y C.E. Offler. 1996. Post-sieve element transport of photoassimilates in sink regions. J. Exp. Bot. 47, 1165-1177.

Peralta-Videa, J.R.; M.L. Lopez; M. Narayan; G. Saupe y J. Gardea-Torresdey. 2009. The biochemistry of environmental heavy metal uptake by plants: Implications for the food chain. Int. J. Biochem. Cell Biol. 41, 1665-1677.
Perfus-Barbeoch, L.; N. Leonhardt; A. Vavasseur y C. Forestier. 2002. Heavy metal toxicity: cadmium permeates through calcium channels and disturbs the plant water status. Plant J. 32(4), 539-548.

Poschenrieder, C. y J. Barceló. 2004. Water relations in heavy metal stressed plants. pp. 249-263. En: Prasad, M. (ed.). Heavy metal stress in plants from biomolecules to ecosystems. 2a ed. Springer-Verlag, New York, NY.

Quigley, F.; J.M. Rosenberg; Y. Shachar-Hill y H.J. Bohnert. 2001. From genome to function: the Arabidopsis aquaporins. Genome Biol. 3, 1-17.

Romero-Puertas, M.C.; M. Rodríguez-Serrano; F.J. Corpas y L.A. del Río. 2004. Cadmium-induced subcellular accumulation of $\mathrm{O}_{2} \cdot-$ and $\mathrm{H}_{2} \mathrm{O}_{2}$ in pea leaves. Plant Cell Environ. 27, 1122-1134.

Ryser, P. y P. Emerson. 2007. Growth, root and leaf structure, and biomass allocation in Leucanthemum vulgare Lam. (Asteraceae) as influenced by heavy-metalcontaining slag. Plant Soil 59, 2461-2467.

Sakurai, J.; F. Ishikawa; T. Yamaguchi; M. Uemura y M. Maeshima. 2005. Identification of 33 rice aquaporin genes and analysis of their expression and function. Plant Cell Physiol. 46, 1568-1577.

Santala, K.R. y P. Ryser. 2009. Influence of heavy-metal contamination on plant response to water availability in white birch, Betula papyrifera. Environ. Exp. Bot. 66, 334-340.

Santoni, V.; L. Verdoucq; N. Sommerer; J. Vinh; D. Pflieger y C. Maurel. 2006. Methylation of aquaporins in plant plasma membrane. Biochem. J. 400, 189-197.

Schroeder, J.I.; G.J. Allen; V. Hugouvieux; J.M. Kwak y D. Waner. 2001. Guard cell signal transduction. Annu. Rev. Plant Physiol. Plant Mol. Biol. 52, 627-658.

Shaw, B.P.; S.K. Sahu y R.K. Mishra. 2004. Heavy metal induced oxidative damage in terrestrial plants. In: Prasad, M. (ed.). Heavy metal stress in plants from biomolecules to ecosystems, second edition. Springer-Verlag, New York, NY.

Siefritz, F.; M.T. Tyree; C. Lovisolo; A. Schubert y R. Kaldenhoff. 2002. PIP1 plasma membrane aquaporins in tobacco: from cellular effects to function in plants. Plant Cell 14, 869-876.

Singh, S. y S. Sinha. 2004. Scanning electron microscopic studies and growth response of the plants of Helianthus annuus L. grown on tannery sludge amended soil. Environ. Int. 30, 389-395.

Sun, M.H.; W. Xu; Y.F. Zhu; W. Su y Z.C. Tang. 2001. A simple method for in situ hybridization to RNA in guard cells of Vicia faba L.: The expression of aquaporins in guard cells. Plant Mol. Biol. Rep. 19, 129 135. 
Tamás, L.; B. Bocová; J. Huttová; I. Mistrík y M. Ollé. 2006. Cadmium induced inhibition of apoplastic ascorbate oxidase in barley roots. Plant Growth Regul. 48, 41-49.

Tamás, L.; J. Dudíková; K. Durceková; J. Huttová; I. Mistrík y V. Zelinová. 2008. The impact of heavy metals on the activity of some enzymes along the barley root. Environ. Exp. Bot. 62, 86-91.

Vander Willigen, C.; O. Postaire; C. Tournaire-Roux; Y. Boursiac y C. Maurel. 2006. Expression and inhibition of aquaporins in germinating Arabidopsis seeds. Plant Cell Physiol. 47, 1241-1250.

Yang, H.M.; X.Y. Zhang y G.X. Wang. 2004. Effects of heavy metals on stomatal movements in broad bean Leaves. Russ. J. Plant Physiol. 51(4), 464-468.

Yang, H.M.; Y. Li y G.X. Wang. 2002. Functions and roles of the channels in broad bean stomatal movements. Acta Phytoecol. Sinica. 26, 656-660.

Yu, Q.; Y. Hu; J. Li; Q. Wu y Z. Lin. 2005. Sense and antisense expression of plasma membrane aquaporin BnPIP1 from Brassica napus in tobacco and its effects on plant drought resistance. Plant Sci. 169, 647-656.

Yukutake, Y.; S.Tsuji; Y. Hirano; T. Adachi; T. Takahashi; K. Fujihara; P. Agre; M. Yasui y M. Suematsu. 2008.
Mercury chloride decreases the water permeability of aquaporin-4-reconstituted proteoliposomes. Biol. Cell. 100(6), 355-363.

Zhang, W. y S.D. Tyerman. 1999. Inhibition of water channels by $\mathrm{HgCl}_{2}$ in intact wheat root cells. Plant Physiol. 120, 849-857.

Zhang, Y.; Z. Wang; T. Chai; Z. Wen y H. Zhang. 2008. Indian mustard aquaporin improves drought and heavy-metal resistance in tobacco. Mol. Biotechnol. 40, 280-292.

Zhang, Z.Q.; W.S. Shu; C.Y. Lan y M.H. Wong. 2001. Uptake and translocation of heavy metals in dominant plants of soil seed banks introduced to a lead/zinc mine tailings pond. Acta Phytoecol. Sinica 25, 306 311.

Zhu, T.; R.L. O’Quinn; W.J. Lucas y T.L. Rost. 1998. Directional cell to cell communication in the Arabidopsis root apical meristem. II. Dynamics of plasmodesmata formation. Protoplasma 204, 84-93.

Zimmermann, H.M.; K. Hartmann; L. Schreiber y E. Steudle. 2000. Chemical composition of apoplastic transport barriers in relation to radial hydraulic conductivity of corn roots (Zea mays L.). Planta 210, 302-311. 